\title{
Recovery from sexual satiety in male rats
}

\author{
SALLY BOLCE JACKSON and DONALD A. DEWSBURY \\ University of Florida, Gainesville, Florida 32611
}

\begin{abstract}
In order to describe more completely the time course of recovery from sexual satiety in male rats, 18 males were tested after each of six recovery intervals: $3,6,9,11,13$, and 15 days. Different recovery rates were found for different measures of behavior. While most measures were restored to baseline levels by 6 days following satiation, the postejaculatory interval following the second ejaculation was still prolonged after 9 days of rest. No changes were seen in the number of intromissions preceding ejaculation. Systematic changes over the course of the experiment were found in the measures of copulatory behavior taken in the sessions that preceded each recovery test.
\end{abstract}

Nearly 40 years ago, Stone and Ferguson (1940) addressed the problem of recovery from sexual satiety in male rats. They concluded: "The period for recovery from copulatory satiation is much longer than that for either food or water satiation. Data now at hand suggest that from approximately 4 to 6 days (possibly more) are required for recovery from a 2 hour period of unrestricted copulation to the degree that the average copulatory frequency on the second two hour period will closely approximate that of the first. The rate of recovery has not yet been fully worked out. This fact has important implications for studies of, or for the interpretation of studies in, sexual drive when the tests follow soon after copulatory satiation" (pp. 429-431). In the ensuing years, there have been several studies of recovery from sexual satiety in male rats; however, despite the obvious importance of knowledge of the time course of recovery to both theory construction and the practical design of experiments, there has yet to appear an adequate delineation of the functional relationship between time and recovery.

Stone and Ferguson (1940) compared copulatory performance on two 2-h tests separated by $24 \mathrm{~h}$. There was no attempt made either to enforce a uniform satiety criterion for all tests or to delineate a systematic functional relationship. Similarly, Larsson (1956) studied recovery, but used 1-h tests with no uniform satiety criterion. Schwartz (1956) studied performance in a situation in which males pressed a bar in order to gain access to a female. He compared performance after 1, 3, and 6 days of

This report is based on a dissertation submitted by the senior author in partial fulfillment of the requirements for the PhD degree at the University of Florida. Research was supported by Grant BMS75-08658. We thank Knut Larsson for aid in designing this study and B. D. Sachs for comments on an initial draft of this manuscript. Hormones were provided through the courtesy of the Schering Corporation, Bloomfield, New Jersey. Reprint requests should be addressed to $D$. A. Dewsbury, Department of Psychology, University of Florida, Gainesville, Florida 32611. recovery from satiety. Folman and Drori (1969) reported that ejaculation frequency was significantly higher after 3 than after 2 days of recovery from satiety and that it was higher after 7 days than after 2 or 3. Karen and Barfield (1975) compared the performance of eight males after 3,6 , and 14 days recovery. Although uniform satiety criteria were used in each of the last three studies, in none was the recovery function studied at enough points to permit systematic delineation of the functional relationship.

The most comprehensive available study of recovery from satiety in rats is that of Beach and Jordan (1956). According to the original design of the Beach and Jordan study, male rats were to be permitted to copulate after recovery periods of 1 , 3 , and 6 days. Twelve animals were tested, using a uniform satiety criterion and different running orders for different individuals. During the course of this experiment, the authors came to believe that recovery might not be complete after the 6-day intervals. Therefore, after the completion of the experiment as designed, additional tests were conducted. Results of all tests were pooled for presentation. Beach and Jordan concluded that recovery from sexual satiety was not complete after 6 days: "As measured by ejaculation frequency, the curve of sexual recovery is negatively accelerated and probably reaches asymptote after 7 to 10 days of rest"' (p. 121).

Although the Beach and Jordan study has become the standard reference used in both experimental design and in model construction, it has at least four major flaws. First, while the initial plan called for a counterbalanced design, the final data included values determined at varying times during the experiment. Second, recovery from satiety may have been incomplete during the baseline tests that occurred 6 days after previous sexual activity. Such effects can be cumulative (Larsson, 1956). Third, Beach and Jordan included only a few scattered 
data points in the critical 7-14-day range. Finally, no inferential statistics were reported.

The objective of the present study was to attempt to delineate the functional relationship describing recovery from sexual satiety more precisely than had been done in previous work.

\section{METHOD}

\section{Subjects}

The subjects were 24 male Long-Evans rats purchased from Blue Spruce Farms, Altamont, New York. They were selected, according to the criteria given below, from a pool of 32 males with no prior copulatory experience. Mean age was 98 days at the time of the first satiety session and 240 days at the time of the last test.

The rats were housed individually in $17.8 \times 25.4 \times 17.8 \mathrm{~cm}$ Wahmann hanging cages in the room in which they were tested. A 12:12 light-dark cycle prevailed, with lights off at $1100 \mathrm{~h}$ and on at $2300 \mathrm{~h}$. Purina Lab Chow and water were continuously available.

Forty-eight females of the same age and strain were maintained in group cages in the same room and were fed the same diet. Eight groups of six females were rotated for use in mating tests throughout the experiment.

\section{Apparatus}

Tests were conducted in four cylindrical Plexiglas arenas, $66 \mathrm{~cm}$ high and $84 \mathrm{~cm}$ in diameter. These were placed on wood platforms, and the floors covered with absorbent litter material. Observations were recorded manually on an Esterline-Angus event recorder.

\section{Procedure}

Preliminary handling. At approximately 80 days of age, the males were earpunched for identification. On each of the next 5 days, each male was allowed to remain alone in an observation arena for $10-15 \mathrm{~min}$.

Test procedures. Tests were conducted in dim light during the last third of the dark phase of the light-dark cycle. Each male was given 3-5 min in the arena before a female was introduced. In satiety tests, males copulated ad lib to the satiety criterion of $30 \mathrm{~min}$ without intromission. In the event that no intromission occurred in the first $15-20 \mathrm{~min}$ of the test, the test was terminated.

Females were brought into behavioral estrus for copulatory tests with intramuscular injections of $0.1 \mathrm{mg}$ estradiol benzoate in oil approximately $74 \mathrm{~h}$ before testing and $1.0 \mathrm{mg}$ progesterone in oil $3-4 \mathrm{~h}$ before testing. Only those females exhibiting good receptivity when checked with a nonexperimental male immediately before a test were used in actual testing.

Pretesting. Pretesting was begun after completion of preliminary handling. Each male was given one to three opportunities to mate with a receptive female. Pretests were terminated after the second ejaculation or after $15 \mathrm{~min}$ if copulation did not take place. A male that did not achieve intromission within $15 \mathrm{~min}$ was given a second pretest 2 days later. Some of those that failed to copulate both times were given a third opportunity several days later. In this way, 24 males were selected as subjects on the basis of having demonstrated successful copulation. Each male then was allowed to copulate to satiety 2 weeks prior to the start of the experiment in order to insure stabilization of the effects of experience.

Design. Copulatory behavior was observed after various periods of recovery from sexual exhaustion. The exhaustion from which recovery was measured was established in a nonexperimental satiety session preceding each experimental test. Thus, each experimental condition actually involved two sessions-one to estab- lish satiety and the other, a certain number of days later, to measure recovery.

The experimental tests were conducted after 3,6,9, 11, 13, or 15 days of recovery. Each subject was tested under each of these conditions. Subsequent to the initial satiety experience, each male copulated to satiety 12 times in the course of the experiment. The experimental tests and the satiety sessions preceding them were identical in every respect except for the length of the rest interval since previous sexual activity. The period elapsing after a test before the nonexperimental satiety session preceding the next test was the standard 2-week period normally allowed for full recovery from satiety.

Six groups of four subjects received the six experimental conditions in different orders. A randomly selected 6 by 6 Latin square provided a randomized, counterbalanced running order, so that each condition appeared first for one group, second for another, and so on (Winer, 1971, p, 693).

A testing schedule was devised to fulfill the requirements of the six groups in such a way that the 312 satiety tests were conducted in the space of 5 months.

\section{Measures}

Copulatory behavior of rats is comprised of bouts of mounts (without vaginal penetration), intromissions (with insertion but no sperm transfer), and ejaculations (with both insertion and sperm transfer). A series is defined as a bout of copulatory activity culminating in an ejaculation and separated from other series (if any) by a postejaculatory refractory period.

A nuinber of measures of copulatory behavior have become standard: mount latency (ML) - the number of seconds from introduction of the female to the first mount, with or without intromission; intromission latency (IL) - the number of seconds from the introduction of the female to the first intromission; ejaculation latency (EL) - the time in seconds from the first intromission of a series to the ejaculation; mount frequency (MF)the number of mounts (without intromission) in a series; in tromission frequency (IF)-the number of intromissions in a series; the mean intromission interval (MIII)-the mean interval separating the intromissions of a series, derived by dividing EL by IF; the postejaculatory interval (PEI)-time in seconds from an ejaculation to the first intromission of the next series; and ejaculation frequency (EF)-the number of ejaculations attained in the test. The series to which a given measure refers can be denoted by appending the number of that series with a hyphen. For example, IF-2 refers to the number of intromissions in the second series. PEI is designated by the number of the series which it follows.

\section{RESULTS}

Data were analyzed for 18 subjects that copulated under each experimental condition. (Four of the original 24 males died before completion of the experiment and two others occasionally failed to copulate.) Measures analyzed included EF, ML, and IL data from each test; EL, IF, MF, and MIII from the first three series of each test, and PEI from the first two series of each test. This was the maximum amount of information that could be analyzed with all males and all tests represented, because males completed varying numbers of series before reaching satiety. Mean values for each measure as a function of recovery interval are presented in Table 1.

Data from all measures except ML and IL were subjected to least squares analyses of variance with 
Table 1

Mean Values and Results of Inferential Statistics for Various Measures of Copulatory Behavior as a Function of Recovery Interval

\begin{tabular}{|c|c|c|c|c|c|c|c|}
\hline \multirow[b]{2}{*}{ Measure } & \multicolumn{6}{|c|}{ Recovery Interval (Days) } & \multirow[b]{2}{*}{$\mathrm{F}$} \\
\hline & 3 & 6 & 9 & 11 & 13 & 15 & \\
\hline ML†† & $22.7^{* *}$ & 11.7 & 47.3 & 10.7 & 11.2 & 10.7 & $\mathrm{~T}=12.8 *$ \\
\hline ILt† & 58.4 & 22.2 & 67.7 & 44.1 & 56.2 & 19.0 & $\mathrm{~T}=7.3$ \\
\hline EF & $4.1 \dagger$ & 5.9 & 6.7 & 6.4 & 6.3 & 6.3 & $6.12+$ \\
\hline EL-1†† & $440.8^{*}$ & 268.4 & 329.9 & 280.8 & 375.8 & 318.7 & $2.90^{*}$ \\
\hline$E L-2 \dagger \dagger$ & $370.1 \dagger$ & 147.1 & 149.3 & 163.2 & 140.6 & 134.7 & $5.95 \dagger$ \\
\hline EL-3†† & $403.4 \dagger$ & 181.5 & 186.7 & 260.2 & 151.1 & 133.5 & $4.88+$ \\
\hline IF-1 & 10.3 & 10.6 & 10.3 & 10.2 & 9.5 & 10.8 & $<1$ \\
\hline IF-2 & 5.4 & 5.1 & 5.2 & 5.3 & 4.7 & 4.6 & $<1$ \\
\hline IF-3 & 5.1 & 4.9 & 4.9 & 5.4 & 5.2 & 4.7 & $<1$ \\
\hline MF-1 & 8.7 & 5.0 & 5.1 & 5.0 & 6.1 & 6.2 & 1.47 \\
\hline MF-2 & $5.2^{*}$ & 2.4 & 2.1 & 2.7 & 1.9 & 3.1 & $3.78 * *$ \\
\hline $\mathrm{MF}-3$ & $6.5^{* *}$ & 3.4 & 3.6 & 4.6 & 1.9 & 1.9 & $3.06^{*}$ \\
\hline MIII- $1+\dagger$ & 44.8 & 26.1 & 33.0 & 30.9 & 36.3 & 43.8 & 1.22 \\
\hline MIII- $2 \dagger \dagger$ & $55.5 \dagger$ & 29.4 & 28.8 & 32.7 & 29.4 & 27.2 & $10.58 \dagger$ \\
\hline MIII-3†† & $78.3 \dagger$ & 38.5 & 36.0 & 42.8 & 31.5 & 29.7 & $7.12 \dagger$ \\
\hline PEI-1 $+\dagger$ & $473.2 t$ & 320.2 & $310 . ?$ & 299.1 & $369.7^{*}$ & 301.8 & $7.38 \dagger$ \\
\hline PEI-2†† & $639.0 \dagger$ & $409.0 \dagger$ & $394.4 \dagger$ & 378.0 & 301.8 & 368.8 & $30.49 \dagger$ \\
\hline
\end{tabular}

$* p<.05 \quad * *<<.01 \quad$ tp $<.001 \quad$ ttIn seconds.

repeated measures, yielding $F$ ratios for effects of groups (i.e., running orders), recovery intervals, and the Group by Interval interaction (Barr \& Goodnight, 1972; Cohen, 1968). Individual comparisons were made using the ratio of the difference between means to the standard error of the difference (Bruning \& Kintz, 1968, p. 113; Winer, 1971, p. 603). Because the distributions for ML and IL were extremely skewed, these scores were analyzed using the Friedman test, a two-way analysis of variance by ranks (Conover, 1971). Individual comparisons were made using a further application of the Friedman test (Conover, 1971). Results of these statistical tests are summarized in Table 1 and were reported in full by Jackson (1975).

No significant effects were found in any of the measures for groups (running orders) or Groups by Recovery Interval interactions.

Ejaculation frequency often is used as a primary measure of recovery from sexual satiety (e.g., Beach \& Jordan, 1956; Folman \& Drori, 1969). EF varied as a function of recovery interval, being significantly lower after 3 days' rest than after the maximum rest period of 15 days (see Table 1). Although the value for EF was still slightly depressed after 6 days, the difference between that value and the Day 15 level was not significant.

Results for the remaining measures generally ran parallel to those for EF. The mean mount latency (ML) after 3 days' rest was significantly longer than that after 15 days of rest, although there was no significant effect of recovery interval on intromission latency (IL).

The mean ejaculation latency (EL) for each of the first three series showed significant variation as a function of recovery interval, with the values on Day 3 significantly above those on Day 15 of recovery.

There was no significant effect of recovery interval on intromission frequency (IF) in any of the first three series.

Although there was no significant effect of recovery interval on mount frequency in the first series, both MF-2 and MF-3 were significantly lower after 3 days' rest than after 15 days.

The pattern of results for mean inter-intromission interval (MIII) was identical to that for MF, with a significant effect in the second and third series, but not the first.

There was a significant effect of recovery interval on postejaculatory interval. PEI-1 was significantly longer on Day 3 than on Day 15. The data from PEI-1 provided the lone exception to the general rule that once a measure recovered to baseline levels it remained there. Although PEI-1 did not differ significantly from Day 15 on Days 6,9 , or 11 , it did so on Day 13. Because the variability of Day 13 scores was unusually high $(\mathrm{SEM}=55.7)$ and the mean was elevated by one atypically high score, the importance of this occurrence is not clear. One may question whether departures from the assumptions underlying the statistical test may have undermined its validity. Indeed, 9 of the 18 males had higher first-series PEIs on Day 15 than on Day 13. Application of the nonparametric Friedman test to these data yielded a nonsignificant result, $T=0.56$; $\mathrm{p}>.05$.

The sole evidence of an orderly effect of recovery intervals longer than 3 days was found in PEI-2. Values of PEI-2 after 3, 6, and 9 days of recovery 
Table 2

Mean Values for Various Measures of Copulatory Behavior for the First and Last Nonrecovery Test and Results of Analysis of Variance

\begin{tabular}{lrrl}
\hline Measure & Test 1 & Test 7 & \multicolumn{1}{c}{ F } \\
\hline EF & 8.7 & 5.6 & $9.30^{* *}$ \\
EL-1 $\dagger$ & 344.4 & 489.6 & $2.30^{*}$ \\
EL-2 $\dagger$ & 142.1 & 224.3 & $2.30^{*}$ \\
EL-3† & 236.8 & 376.8 & 1.54 \\
IF-1 & 12.7 & 11.1 & $2.72^{*}$ \\
IF-2 & 5.3 & 5.6 & $<1$ \\
IF-3 & 6.5 & 6.7 & 1.03 \\
MF-1 & 4.8 & 6.3 & 1.22 \\
MF-2 & 1.6 & 3.9 & $2.40^{*}$ \\
MF-3 & 4.4 & 7.9 & 1.71 \\
MIII-1 $\dagger$ & 27.2 & 44.6 & 1.68 \\
MIII-2 $\dagger$ & 27.5 & 39.3 & $2.26^{*}$ \\
MIII-3 $\dagger$ & 30.9 & 56.7 & $2.76^{*}$ \\
PEI-1 $\dagger$ & 326.1 & 368.7 & 1.87 \\
PEI-2 $\dagger$ & 388.8 & 403.9 & 1.45 \\
\hline
\end{tabular}

Note-F ratios were calculated using all seven nonrecovery tests. $* p<.05 \quad * *<.0001 \quad$ tIn seconds.

were significantly higher than those after 15 days of rest.

Each male received a total of seven additional satiety tests-one initial pretest and a test before each recovery test-from which the data have not yet been presented. These tests were spaced over a total of 5 months. A search was made for any trends occurring over that period. Means were calculated for the various measures in each of the seven nonexperimental tests and data were subjected to analyses of variance. Mean scores for the first and last of these nonrecovery tests and results of analyses of variance are presented in Table 2 . Significant differences were found for 7 of the 15 measures considered. There was a significant decrease in IF-1 across tests. All other changes were in the direction that would indicate a retardation rather than facilitation of copulatory activity (i.e., longer latencies or intervals and more mounts preceding ejaculation). For most of the measures showing significant effects, the differences occurred near the end of the 5-month period, but EF showed decreases in each successive test from the first to the last (see Jackson, 1975).

\section{DISCUSSION}

The present data are the most substantial and systematic of any study of recovery from sexual satiety yet conducted and permit a more accurate characterization of the time course of this process than has been heretofore possible. Recovery from sexual satiety appears more rapid than previously considered (e.g., Beach \& Jordan, 1956). Copulatory behavior was substantially depressed when male rats mated with just 3 days' rest following sexual satiety.
The males took longer to achieve their first mount and attained fewer ejaculations than in the rested condition. The time course of copulatory activity was generally slowed, with increases apparent in ejaculation latency in the first three series, mean inter-intromission interval in the second and third series, and postejaculatory interval following the first two series. More mounts preceded ejaculation in the second and third series. There were no changes in the number of intromissions preceding ejaculation.

After rest periods of 6 or 9 days, all measures considered were restored to values near those characteristic of the rested condition, with the exception of the postejaculatory interval in the second series. The only significant difference from the rested condition found in males with 11 or 13 days rest was an anomalous effect on PEI-1 at Day 13.

It is apparent that there is no single time at which one can consider a male rat to be recovered from sexual satiety; the recovery time will vary with the criteria adopted for both satiation and recovery. If one adopts a criterion related to ejaculation frequency or to the various measures of behavior througn the occurrence of a second ejaculation, recovery is essentially complete after 6 days. Recovery of all measures presented in Table 1 is complete by Day 11. Should one be interested only in intromission frequency, recovery might be regarded as complete by Day 3.

Although previous studies of recovery from sexual satiety have been less comprehensive than the present study, data from all studies are generally consistent with respect to the relative recovery rates of different aspects of the total pattern. Thus, pronounced increases in PEI during recovery have been reported by Beach and Jordan (1956), Karen and Barfield (1975), and Larsson (1956). Reductions in EF were reported by Beach and Jordan (1956), Folman and Drori (1969), and Karen and Barfield (1975); Larsson's data on EF are not comparable as they are based on time-limited tests. Both Karen and Barfield (1975) and Larsson (1956) found IF to be rather stable throughout sexual recovery, as in the present data. The data from Beach and Jordan (1956) are an apparent exception to the uniform finding of stability with respect to IF, as they reported a higher IF on Day 3 than on either Day 6 or Day $15(8.2,6.2$, and 6.3, respectively). Examination of these data suggests that this discrepency may be more apparent than real. Values reported by Beach and Jordan were averaged across all series. Males normally require more intromissions in the first than in later series. Since the males attained fewer ejaculations on Day 3, the relative weight of data from the first series would be much greater than in rested males. Thus, the apparent increase in IF is probably a mere function of the increased 
weighting of the first series as a consequence of the decreasing number of ejaculations. In fact, when the "copulations per ejaculation" figure of Beach and Jordan is calculated using their fully rested IF values and the Day $3 \mathrm{EF}$ value, a score of 8.3 is obtained.

As in the present study, other workers have found effects of recovery on behavior within ejaculatory series to be smaller and less consistent than those on EF and PEI. In the present study, such effects were more pronounced in the second and third than in the first series. A similar sensitivity of measures of later series behavior to experimental treatments has been noted in studies of effects of copulatory experience and drug administration (Dewsbury, 1969, 1971; Dewsbury \& Davis, 1970).

It should be noted that, in the present study and in the studies of both Beach and Jordan (1956) and Karen and Barfield (1975), small and generally statistically insignificant depressions of EF occurred on Day 6. It remains possible that this is a true difference of small magnitude which cannot be detected reliably with available sample sizes and the power of available statistical tests (Cohen, 1969). Folman and Drori (1969) did detect a significant difference between 2 or 3 days of recovery and 7 days.

These data are relevant to the construction of theoretical models of the control of copulatory behavior. Beach and Jordan (1956) proposed that copulatory activity is a reflection of the operation of two separate mechanisms: an arousal mechanism (AM) that "mediates the awakening and increase of sexual excitement which eventuates in copulation" (p. 131) and a copulatory-ejaculatory mechanism (CM) which mediates mounting and intromission. Measures such as ML, IL, and PEI were hypothesized as regulated by the AM, whereas EL and IF were thought to be controlled by the $C M$. Since the proposal of the original model, other workers have modified and expanded it, usually by introducing additional hypothetical mechanisms (e.g., Karen \& Barfield, 1975; McGill, 1965; Sachs \& Barfield, 1974, 1976).

The pattern of recovery from satiety appears to form three clusters of measures which recover differentially: IF is unaffected, PEI is slow to recover, and ML, MIII, EL, and MF are moderately affected. This recovery pattern is inconsistent with various aspects of the original model. While ML, IL, and PEI were hypothesized as reflicting activity in the AM, their differential recovery rates suggest different control mechanisms. Similarly, it is difficult to reconcile the differential effects of recovery on EL and IF with the original model. More recent models, of the sort anticipated by Beach (1956), have been based on more mechanisms and an increased number of measures (see Sachs \& Barfield, 1976).
For example, following the description of ultrasonic vocalization in the PEI (Barfield \& Geyer, 1975), some authors have divided the PEI into an absolute and relative refractory period using the ultrasonic call as an indicator. Although these distinctions had not been made at the time the present data were collected, models based on these concepts may contain enough flexibility to account for the results.

Sachs (1978) has conducted a factor analysis of the interrelatedness of different measures of copulatory behavior. Interestingly, IF was found to be virtually independent of all other measures. That same independence is confirmed in the present data. Although the patterns of intercorrelations of several other clusters of measures in the factor analysis differ somewhat from the clusters observed during recovery, these differences may be explainable as resulting from overlapping and interacting mechanisms.

An unexpected finding in the present study was that copulatory behavior in fully rested animals changed over the course of the experiment. A general retardation of behavior was reflected in increased ELs, MIIIs, and MFs, and decreased EFs. By contrast, IF-1 was decreased. There are several possible explanations. First, the rats aged from 3 to 8 months over the course of this experiment and age per se could have produced these changes. Although changes in copulatory behavior have been reported as a function of age (Larsson, 1956; Larsson \& Essberg, 1962), they are generally in the direction opposite to those in the present study. Second, since each male copulated to satiety a total of 13 times in the course of this experiment, experience could have produced the effect. Effects of experience on copulatory behavior have been demonstrated (Dewsbury, 1969; Larsson, 1959), but they have generally been small and often in a direction opposite to the present effects. Third, although the animals were kept under a regime of regular photoperiodicity and relatively constant temperature conditions, it is possible that the effect is the result of seasonal changes. Thus, various species, as for example whitefooted mice, kept under similar conditions, show a seasonal cycle of gonadal function in the laboratory (Bartke \& Chang, 1973). Finally, it is possible that, while 15 days is sufficient to produce full recovery as assessed by the measures recorded herein, some more subtle cumulative effect of repeated testing may build up and produce a progressive retardation of behavior. Although there were progressive changes in baseline performance as testing proceeded, the use of randomization procedures distributed this effect equally across conditions and minimized effects on the data on recovery from satiety, the phenomenon of primary interest.

One question that remains is that of why recovery 
from sexual satiety should take so long compared to recovery in other motivational systems. One clue may come from a recent study of the replenishment of the secretions of the seminal vesicles after copulation (Pessah \& Kochva, 1975). Secretions are stored in the lumina of the seminal vesicle. The vesicles are virtually emptied of their secretions after a prolonged series of copulations. Pessah and Kochva found that the luminal secretions increase continuously and "at the last stage measured in this series $(26 \mathrm{~h})$, it is still only one third of that found in the virgins. Since after one week it is completely replenished, the end of the process should probably be somewhere in between 3 and 7 days" (p. 560). If copulation during a period in which the vesicles were relatively empty would reduce the likelihood of successful pregnancy initiation, it would seem reasonable that the mechanisms controlling behavior might have evolved in consort with those controlling vesicular secretory activity. The time courses of these two processes appear quite parallel. Although this parallelism does not prove any relationship, it suggests a direction for future research.

\section{REFERENCES}

Barfield, R. J., \& Geyer, L. A. The ultrasonic postejaculatory vocalization and the postejaculatory refractory period of the male rat. Journal of Comparative and Physiological Psychology, 1975, 88, 723-734.

BARr, A. J., \& Goodnight, J. H. Statistical analysis system. Raleigh, N.C: North Carolina State University, 1972.

BARTKE, A., \& Chang, M. C. Seasonal variation in male gonadal function in a laboratory population of Peromyscus leucopus. American Midland Naturalist, 1973, 89, 490-495.

ВЕАСH. F. A. Characteristics of masculine "sex drive." In M. R. Jones (Ed.), Nebraska symposium on motivation. 1956. Lincoln: University of Nebraska Press, 1956. Pp. 1-32.

Beach. F. A., \& Jordan, L. Sexual exhaustion and recovery in the male rat. Quarterly Joumal of Experimental Psychology, 1956, 8, 121-133.

Bruning, J. L., \& Kintz, B. L. Computational handbook of statistics. Glenview, Ill: Scott, Foresman, 1968.

CoHen, J. Multiple regression as a general data-analytic system. Psychological Bulletin, 1968, 70, 426-443.

CoHen, J. Statistical power analysis for the behavioral sciences. New York: Academic Press, 1969.

Conover, W. J. Practical nonparametric statistics. New York: Wiley, 1971.
Dewsbury, D. A. Copulatory behavior of rats (Rattus norvegicus) as a function of prior copulatory experience. Animal Behaviour. $1969.17,217-223$.

Dewsbury, D. A. Copulatory behavior of rats following reserpine administration. Psychonomic Science, 1971, 22, 177.179.

Dewsbury, D. A., \& Davis, H. N. Effects of reserpine on the copulatory behavior of male rats. Physiology \& Behavior, 1970. 5. 1331-1333.

Folman, Y.. \& Drori, D. Effects of the frequency of mating on the androgen-sensitive organs and sexual behavior. Physiology \& Behavior, 1969, 4, 1023-1026.

JACKSON, S. B. Recovery from sexual satiety in male rats. Doctoral dissertation, University of Florida, 1975.

Karen, L. M., \& Barfield, R. J. Differential rates of exhaustion and recovery of several parameters of male rat sexual behavior. Journal of Comparative and Physiological Psychology, 1975, 88, 693-703.

LARsSON, K. Conditioning and sexual behavior in the male albino rat. Stockholm: Almqvist \& Wiksell, 1956.

LARSSON, K. Experience and maturation in the development of sexual behavior in male puberty rat. Behaviour, 1959, 14, 101-107.

Larsson, K., \& Essberg, L. Effect of age on the sexual behavior of the male rat. Gerontologia Clinica, 1962, 6, 133-143.

McGill, T. E. Studies of the sexual behavior of male laboratory mice: Effects of genotype, recovery of sex drive, and theory. In F. A. Beach (Ed.), Sex and behavior. New York: Wiley, 1965. Pp. 76-88

Pessah, H., \& Kochva, E. The secretory activity of the seminal vessicles in the rat after copulation. Biology of Reproduction, $1975,13,557-560$.

SACHS, B. D. Conceptual and neural mechanisms of masculine copulatory behavior. In T. E. McGill, D. A. Dewsbury, \& B. D. Sachs (Eds.), Sex and behavior: Status and prospectus. New York: Plenum, 1978. Pp. 267-295.

SACHS, B. D., \& BARFIELD, R. J. Copulatory behavior of male rats given intermittent electric shocks: Theoretical implications. Journal of Comparative and Physiological Psychology, 1974, 86, 607-615.

SACHS, B. D., \& BARFIEld, R. J. Functional analysis of masculine copulatory behavior in the rat. In J. S. Rosenblatt, R. A. Hinde, E. Shaw, \& C. Beer (Eds.), Advances in the study of behavior (Vol. 7). New York: Academic Press, 1976. Pp. 91-154.

SchWARTz, M. Instrumental and consummatory measures of sexual capacity in the male rat. Journal of Comparative and Physiological Psychology, 1956, 49, 328-333.

Stone, C. P., \& Ferguson, L. W. Temporal relationships in the copulatory acts of adult male rats. Journal of Comparative Psychology, 1940, 30, 419-434.

WINER, B. J. Statistical principles in experimental design (2nd ed.). New York: McGraw-Hill, 1971.

(Received for publication July 1, 1977; revision accepted February 8, 1978.) 